\title{
Kernos
}

Revue internationale et pluridisciplinaire de religion grecque antique

$31 \mid 2018$

Varia

De la crise naquirent les cultes. Approches croisées de la religion, de la philosophie et des représentations antiques

\section{Alaya Palamidis}

\section{(2) OpenEdition}

1 Journals

\section{Édition électronique}

URL : http://journals.openedition.org/kernos/2946

DOI : $10.4000 /$ kernos.2946

ISSN : 2034-7871

\section{Éditeur}

Centre international d'étude de la religion grecque antique

\section{Édition imprimée}

Date de publication : 1 décembre 2018

Pagination : 329-332

ISBN : 978-2-87562-055-2

ISSN : 0776-3824

\section{Référence électronique}

Alaya Palamidis, " De la crise naquirent les cultes. Approches croisées de la religion, de la philosophie et des représentations antiques », Kernos [En ligne], 31 | 2018, mis en ligne le 01 octobre 2018,

consulté le 25 janvier 2021. URL : http://journals.openedition.org/kernos/2946 ; DOI : https://doi.org/ $10.4000 /$ kernos.2946

Ce document a été généré automatiquement le 25 janvier 2021.

Kernos 


\title{
De la crise naquirent les cultes. Approches croisées de la religion, de la philosophie et des représentations antiques
}

\author{
Alaya Palamidis
}

\section{RÉFÉRENCE}

Marco CAVALIERI, René LEBRUn, Nicolas L. J. MEUNIER (éd.), De la crise naquirent les cultes. Approches croisées de la religion, de la philosophie et des représentations antiques. Actes du Colloque international organisé à Louvain-la-Neuve les 12 et 13 juin 2014 par le Centre d'étude des Mondes Antiques, le Centre d'Histoire des religions Cardinal Julien Ries et les Actions de Recherche Concertées « A World in Crisis? », Turnhout, Brepols, 2015. 1 vol. 15,6 × 23,4 cm, 311 p. (Homo Religiosus, Série II, 15). ISBN : 978-2503-55461-7.

1 Cet ouvrage constitue les actes d'un colloque organisé à l'Université catholique de Louvain les 12 et 13 juin 2014 par le Centre d'Étude des Mondes Antiques, le Centre d'Histoire des Religions Cardinal Julien Ries et les Actions de Recherche Concertées «A World in Crisis? ». Le titre du recueil, « De la crise naquirent les cultes », tout comme celui du colloque, «Cultes en crise, crise des cultes ", reflète imparfaitement le thème commun aux différentes contributions, c'est-à-dire la façon dont une crise peut se répercuter dans le domaine religieux, aussi bien en donnant naissance à de nouveaux cultes - qualifiés de cultes de crise - qu'en entraînant des évolutions ou transformations de certains cultes existants. Une crise est ici définie comme tout changement important, positif ou négatif, qui peut être de nature politique, économique, environnementale ou encore religieuse. L'ouvrage regroupe des 
contributions concernant le Proche-Orient ancien, les mondes grec et romain et l'Antiquité tardive.

2 Ainsi, Jan Driessen s'intéresse à quatre périodes charnières de l'histoire de la Crète minoenne, définies par la construction ou la destruction des palais ainsi que l'éruption du volcan de Santorin. Dans un premier temps, la construction des premiers palais qui entraîne une centralisation de certaines pratiques rituelles semble s'accompagner du développement de sanctuaires de sommets et de grottes, fréquentés par la population rurale. La centralisation s'accentue après la destruction des premiers palais et la construction des seconds puisque les sanctuaires de sommets et de grottes semblent soit récupérés par l'élite palatiale soit, pour les plus lointains, abandonnés, tandis que se répand le type iconographique de la "Grande déesse» qui constituerait une déification de l'ancêtre féminine de la dynastie royale de Knossos. Après l'éruption du volcan de Santorin, l'apparition d'une iconographie masculine, représentée notamment par le kouros de Palaikastro, représenterait une réaction à la centralisation des cultes autour de Knossos. Après la destruction des seconds palais, cette iconographie, qui aurait favorisé l'apparition de Zeus Diktaios sur les tablettes en linéaire B de Knossos, disparaît, comme l'atteste la mutilation volontaire du kouros de Palaikastro. Nous ignorons si Zeus Diktaios cesse d'être honoré entre la disparition de la civilisation mycénienne à Knossos et le début de l'Âge du fer ou si seule sa représentation anthropomorphique a disparu. Quoi qu'il en soit, l'iconographie masculine représentée notamment par le kouros de Palaikastro constitue une parenthèse dans l'histoire cultuelle de la Crète et il semble dès lors possible de parler de « culte de crise " à propos du culte de la divinité masculine qui s'est développé après l'éruption du volcan de Santorin.

3 Charles Doyen étudie la tablette PY Tn 316, rédigée en linéaire B peu avant la destruction définitive du palais de Pylos et qui mentionne des offrandes destinées à diverses divinités dans plusieurs sanctuaires pyliens. En raison du caractère inachevé du texte, de la richesse des offrandes et de la mention d'êtres humains, qui auraient été sacrifiés, certains chercheurs ont émis l'hypothèse qu'il s'agissait de rituels exceptionnels mis en place dans la période trouble qui a précédé la destruction du palais. Au contraire, comme d'autres chercheurs, l'A. y voit un simple calendrier cultuel évoquant des fêtes ayant lieu de façon périodique. Les êtres humains mentionnés constitueraient le personnel du culte plutôt que des personnes sacrifiées, tandis que rien ne permet d'affirmer que les divinités dont la présence sur cette tablette constitue un hapax ont été introduites à cette occasion dans un contexte de crise.

4 Patrick Marchetti s'intéresse au vers attribué par des auteurs chrétiens à Épiménide, qui concerne « les Crétois toujours menteurs, bêtes méchantes, ventres paresseux ». Au moyen d'une comparaison avec un vers d'Hésiode (Theog. 26) auquel le vers attribué à

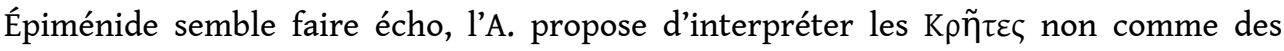
Crétois mais comme des mangeurs de viande, dont le poète dénoncerait le mode de vie et qu'il encouragerait à adopter l'agriculture. C'est de cette mauvaise interprétation du vers dès l'Antiquité que serait née l'hypothèse erronée selon laquelle Épiménide était originaire de Crète et qu'il situait la naissance de Zeus sur cette île. Au contraire, l'A. identifie de nombreux éléments qui peuvent être mis en lien avec le Péloponnèse dans les fragments du poète, ce qui suggérerait selon lui que ce dernier situait la naissance de Zeus en Arcadie. Dans le contexte de la crise qui aboutit à la fondation des poleis, Épiménide aurait ainsi cherché à « convertir [les pasteurs] à un nouveau mode de vie, 
en les plaçant sous l'autorité d'un nouveau dieu, chargé d'établir la synthèse, Zeus " (p.96). Il s'agit d'une hypothèse séduisante mais qui pose de nombreuses questions. Quelles sont par exemple les sources qui permettent d'affirmer que Zeus est un dieu jeune «qui émerge précisément à cette époque par l'action des Sages »(p.87)? Comment une interprétation erronée du vers attribué à Épiménide, selon laquelle les Crétois étaient des menteurs, aurait-elle suffi à faire du poète un Crétois ? Et peut-on vraiment tenter de reconstituer la pensée d'Épiménide à partir d'œuvres « réelles ou apocryphes peu importe» (p.88), alors même que l'attribution à cet auteur du vers dont il est question est tardive?

5 Rachele Dubbini étudie la recomposition du paysage religieux de l'agora de Corinthe après l'importante destruction qui a frappé cet espace dans le premier quart du $\mathrm{VI}^{\mathrm{e}}$ siècle et qui pourrait être attribuée à la révolte contre la tyrannie cypsélide. Audessus de certains édifices en ruines ont alors été construits des "stele shrines", sanctuaires vraisemblablement destinés à honorer les victimes décédées lors de la destruction de ces bâtiments et qui auraient peu à peu acquis le statut d'ancêtres communs à un groupe familial ou une petite communauté. La construction ou reconstruction d'autres édifices aurait été destinée à effacer le souvenir de la dynastie cypsélide et à mettre en avant un passé mythique qui n'aurait pas été exploité par cette dernière. Par exemple, la fontaine "cyclopéenne " près de la source Pirène, délaissée pendant l'époque cypsélide, aurait été monumentalisée en raison de son lien probable avec le mythe de Pégase et Bellérophon. C'est l'intervention d'Athéna Chalinitis dans le même mythe qui expliquerait la construction d'un temple destiné à la déesse à l'emplacement des futurs édifices liés au culte impérial ou capitolin, si l'on accepte l'attribution hypothétique du temple à Athéna et sa fondation au vie siècle plutôt qu'à une date antérieure. L' A. propose ainsi une reconstitution intéressante des facteurs qui ont présidé à la reconstruction de l'espace public, qui, malheureusement, ne peut être vérifiée en raison d'une pénurie de sources à ce sujet.

Christophe Flament fait le point sur l'état des cultes traditionnels et sur l'introduction de cultes de divinités étrangères en Attique pendant la guerre du Péloponnèse et rejette l'affirmation selon laquelle il existerait une "crise des cultes » et des « cultes de crise " à cette époque. Parmi les cultes de nouvelles divinités, dont l'introduction aurait été facilitée, selon une opinion commune, par le fait que les divinités traditionnelles avaient montré leurs limites au cours de la Grande peste, celui de Bendis était déjà implanté en Attique avant le début de l'épidémie et son introduction ne peut dès lors pas être expliquée comme une réaction à celle-ci. Quant au culte d'Asklépios, il ne fut introduit que six ans après la fin de l'épidémie et son implantation en Attique s'expliquerait plutôt par une volonté de favoriser les relations diplomatiques entre Athènes et Épidaure. Par ailleurs, contrairement à l'affirmation de Thucydide, rien ne permet de vérifier que les divinités traditionnelles ont été négligées. Au contraire, des inscriptions témoignent de la volonté de rembourser les dieux des sommes empruntées pour les efforts de guerre et même d'une reprise des travaux de construction sur l'Acropole à deux reprises au cours de la guerre. Ce conflit aurait même favorisé un retour à une religion plus traditionnelle, ce dont témoigne notamment le discours Contre Nicomachos de Lysias, qui attribue les malheurs d'Athènes au manque de respect de la "constitution des ancêtres ", notamment en matière religieuse, ou encore le procès de Socrate, accusé de rompre avec les traditions ancestrales. On regrette ici l'absence presque totale de bibliographie postérieure à 2009 et notamment de deux 
articles de François de Polignac sur l'introduction des cultes d'Amphiaraos et d'Asklépios en Attique $^{1}$, qui auraient été pertinents pour cette étude.

Platon explique lui aussi les problèmes que connaît Athènes au lendemain de la guerre du Péloponnèse par une crise religieuse, mais offre une analyse différente des raisons de cette crise et y propose des solutions dans la législation de ses cités idéales, comme le montre l'article d'Aikaterini Lefka. Le philosophe prend position contre toute doctrine athée ou agnostique, source selon lui d'impiété et par conséquent de manque de respect envers les lois. Il réaffirme pour cela l'existence des dieux en invoquant comme preuves les signes les plus manifestes de leur existence, les corps célestes et l'âme. Platon critique par ailleurs l'anthropomorphisme et le caractère souvent immoral des dieux que véhiculent les mythes, qui ont selon lui une influence néfaste sur la société. À ces mythes, il oppose divers principes théologiques, dont le premier est que les dieux sont bons et qu'ils ne peuvent dès lors pas être à l'origine des maux humains. De ce principe découle le second, selon lequel un dieu ne peut changer d'apparence pour tromper quelqu'un. L'A. explore ensuite la façon dont la croyance aux divinités «traditionnelles » s'accorde avec les théories métaphysiques du philosophe. Selon ce dernier, les divinités du panthéon grec, issues de l'union d'Ouranos et Gaia, eux-même créés par le Démiurge, assurent le bon fonctionnement et l'harmonie du monde. En contemplant périodiquement les Idées, elles deviennent des guides pour les humains et c'est notamment à travers les sciences, la philosophie et les arts qu'elles éduquent les âmes humaines et qu'elles les poussent à être de bons citoyens.

Nicolas Amoroso s'intéresse quant à lui à la persistance de cultes domestiques polythéistes, grecs et romains, dans l'Antiquité tardive christianisée, en prenant l'exemple de groupes de statuettes ou d'autres objets associant des divinités isiaques à d'autres figures divines, en particulier Isis à Tychè/Fortuna, association qui trouve ses origines dans l'Égypte ptolémaïque. Des tels groupes ont notamment été découverts dans ou à proximité de maisons à Athènes, Cos ou encore Corinthe. L'A. précise toutefois que les statuettes sont souvent trouvées en remploi et qu'elles s'accompagnent rarement de traces de sacrifice, si bien qu'il est difficile, dans la plupart des cas, de s'assurer qu'elles avaient une fonction cultuelle et pas simplement décorative.

Comme le souligne Marco Cavalieri dans le débat conclusif, il n'est pas possible d'établir une synthèse et de proposer une typologie des « cultes de crise " puisque les situations de crise ont donné lieu à des réponses très variées dans le domaine religieux. Une conclusion qui ne surprend pas étant donné la très large définition du concept de crise adoptée dans l'ouvrage.

\section{NOTES}

1. «Un système religieux à double visage dans un espace intermédiaire: l'exemple de l'Amphiaraion d'Oropos ", in N. BELAYCHE, J.-D. DuboIs (éd.), L'oiseau et le poisson. Cohabitations religieuses dans les mondes grec et romain, Paris, 2011, p. 93-105; «Entre privé, public, civique : à 
propos de l'intégration des cultes extérieurs dans l'Athènes classique », in B. LEGRAS, G. THÜR (éd.), Symposion 2011. Études d'histoire du droit grec et hellénistique, Vienne, 2012, p. 199-210.

\section{AUTEURS}

ALAYA PALAMIDIS

AnHiMA - EHESS - Paris 